\title{
A Study of Student Satisfaction Index to Logistic Service Provided by Enterprises for Chinese Overseas Higher Education Based on ACSI Model
}

\author{
LUO Xing
}

Institute of Chateauroux, Beijing International Studies University, Beijing 100024, China

luoxing@bisu.edu.cn

Keywords: overseas education; logistic service; student satisfaction index model; PLS

\begin{abstract}
Some Chinese universities are having a tendency to run their schools in overseas countries. When those universities involved offer students logistic support by means of purchasing such services from overseas enterprises, an evaluation system should been established. In this paper, an empirical research is conducted on student satisfaction index to logistic services provided by Pôle d'enseignement supérieur international (PESI), using the parameter to interpret the student satisfaction index based on ACSI model by partial least squares path (PLS). The paper is concluded with some suggestions to have a relatively high student satisfaction index to overseas logistic services: raising awareness of the service to students, improve service quality and providing high cost-effective services to meet the students' needs.
\end{abstract}

\section{基于ACSI模型的高校海外办学中企业提供后勤服务满意度指数模型研究 \\ 骆行 \\ 北京第二外国语学院夏斗湖学院, 北京, 中国 \\ luoxing@bisu.edu.cn}

关键词：海外办学；后勤服务； 满意度指数模型；PLS

摘要：随着中国高等教育走出去, 高校赴海外办学趋势逐步提升。在办学过程中, 高校可以 通过向企业购买服务做好海外校区的后勤保障, 需要建立对后勤服务的评价体系。本研究在 ACSI 模型的基础上, 通过对法国夏斗湖市 PESI 校区提供的后勤服务开展满意度实证调查, 运用 PLS 结构方程拟合、修正和解释, 建立海外校区后勤服务满意度指数模型。研究结果表 明, 要想提升海外校区后勤服务满意度, 应该做好校区后勤服务的宣传工作, 深入了解海外 校区学生的后勤服务需求, 提升服务质量, 提高后勤服务的性价比。

\section{1.引言}

随着中国高等教育走出的趋势, 办学国际化是中国高校发展的必经之路。赴海外办学既 可以利用海外优秀的教学资源, 提高人才培养成效, 还可以提升高校在海外的知名度, 建立 国际合作，是高校国际化的重要途径。

随着维护办学主权、提升管理水平、服务办学和师生生活以及降低办学成本的需要, 建 立自主的后勤保障体系可能成为中国高校的发展目标。然而国内外法律法规、政府政策、社 会风俗等客观环境存在巨大差异, 中国高校在建立海外校区时在后勤管理上可能遭遇文化休 克现象, 对教学活动的开展和师生正常生活造成严重影响; 此外, 完整的后勤保障体系不可 能一蹴而就的建立，因此高校势必会利用当地教育资源或向企业直接购买后勤服务。由此可 
见, 在海外办学过程中建立对后勤服务的满意度评价体系的重要性尤为突出, 高校可以通过 该体系评价供应商后勤服务水平，促进供应商提升服务质量，为购买后勤服务决策提供依据。 然而目前中国高校赴海外办学主要采用合作办学模式, 其后勤服务基本上依托于本土高校或 者当地社会，很少涉及对海外校区的直接管理，对海外校区的后勤保障体系的研究很少，因 此相关研究具备重要性和紧迫性。

本文通过建立学生对法国 PESI 校区提供的后勤服务满意度指数模型, 寻找影响后勤满意 度指标的因素, 帮助高校在海外办学中建立后勤评价体系, 为高校海外办学教学及师生生活 提供更好的保障。

PESI 校区是由 SCZY 集团在法国夏斗湖市投资建设的教育服务实体，向中国高校提供教 学生活场所及后勤服务, 设立 1 栋教师宿舍、 2 栋学生宿舍、1 栋食堂兼自习室、 1 栋教学楼 及 1 个田径体育场, 聘用工作人员 2 人, 其中中国籍管理人员 1 人, 法国籍工勤人员 1 人。 至 2018 年 10 月, 共有 BISU 和 BSU 两所高校的 175 名学生进驻学习。

\section{2.文献回顾}

通过在中国知网上查阅包含 “高校”、“海外办学”、“后勤” “满意度” 等关键词 的论文, 发现近 5 年基本上没有关于高校海外办学中后勤保障的研究; 关于高校后勤服务满 意度的研究也较少, 且定性研究较多, 量化研究很少。其中林文信 (2014) 认为以顾客满意 度为导向, 建立以学生为中心的高校后勤管理模式, 可以有效地改进高校后勤服务的质量和 效率，提高后勤服务的满意度，实现后勤管理服务的创新 ${ }^{[1]}$ 。冯宝军、张思鹏（2014）基于 案例分析，兼顾高校后勤服务外包中普遍存在的产权不清晰及代理风险等问题，构建了基于 分类管理与部分外包的高校后勤服务外包框架 ${ }^{[2]}$ 。贾斌、王玮 (2014) 通过对问卷调查结果进 行因子分析, 得出扬州工业职业技术学院学生对后勤满意度评价体系包括食堂服务、住宿服 务、物业服务、商贸服务和医疗服务 5 个因子，其中满意度最低的是住宿服务因子，最高的 是医疗服务因子 ${ }^{[3]}$ 。王文雅、陈斌斌 (2016) 通过问卷调查得出, 北大医学部师生对后勤服务 最关注的问题主要集中在餐饮、公寓房、宿舍管理、校园环境卫生和出行用车 4 个方面 ${ }^{[4]}$ 。仲 懿、张海军（2017）使用结构方程模型对高职大学生后勤满意评价体系建模，找到对后勤服 务满意度产生正向作用的 5 个因素, 按照影响强度从高到低分别是餐饮服务、维修服务、医 疗服务、物业服务和商贸服务 ${ }^{[5]}$ 。孙涛 (2018) 通过问卷调查的形式, 得出西安某高校师生在 后勤服务中最关心的是日常饮食问题和住宿休息，对身体健康和生活环境的要求比较高，就 餐饮服务、水电暖供应和公寓服务 3 个师生重点关心的项目提出了提升策略 ${ }^{[6]}$ 。

此外, 还有研究者深入研究后勤服务中食堂满意度、公寓满意度等内容。田亮、田文芳 （2015）通过对问卷结果进行因子分析, 得出影响新疆塔里木大学师生对食堂满意度包括食 堂饭菜质量、食堂服务水平、食堂卫生 3 个主要因素 ${ }^{[7]}$ 。刘雪萍 (2014) 通过因子分析和结构 方程模型建模, 得出影响福州市某高校公寓满意度有宿舍整体、公寓位置、公寓清洁、公寓 楼道、维修服务和人员服务 6 个因素，其中学生最关注的是宿舍硬件设施 ${ }^{[8]}$ 。

中国高校在海外自主建设校区的案例很少，前人对中国高校海外后勤保障体系的满意度 评价研究缺乏基础条件。对高校后勤保障体系满意度的研究多为定性研究, 通过总结工作经 验、案例分析等方式探讨后勤保障体系的管理模式及服务框架等内容, 缺少理论依据。部分 研究者基于工作经验, 通过结构方程、因子分析等方式建立了满意度评价模型。然而建立模 型时没有基于普遍承认的理论, 也仅仅考虑了学生对后勤服务内容的满意度, 缺少对其他维 度及相关关系的深入研究。本研究基于 ACSI 模型, 建立影响学生对海外校区后勤服务保障体 系满意度模型, 分析了影响后勤满意度的多个维度及之间的因果关系, 具有一定的创新性和 实用价值。 


\section{3.理论模型构建}

\section{1 模型选用和研究假设}

学生对后勤满意度来源于顾客满意度，指学生对海外校区服务供应商所提供的后勤服务 的直接性综合评价 ${ }^{[9]}$ 。多个国家都建立了自己的顾客满意度评价模型, 其中, 最具代表性的模 型为瑞典顾客满意指数模型 (SCSB)、美国顾客满意指数模型 (ACSI) 和欧洲顾客满意指数 模型（ECSI），其中 ACSI 模型是 Fornell 等人在瑞典顾客满意指数模式（SCSB）的基础上创 建的顾客满意度指数模型, 模型基于消费者的消费认知过程, 将消费者的总体满意度置于因 果互动系统中。该模型可解释消费经过与整体满意度之间的关系, 并能指示出满意度高低将 带来的后果，从而赋予了整体满意度前向预期的特性。

考虑到 ACSI 模型关于学生对后勤满意度的解释度较高, 且将影响后勤满意度的维度纳 入研究, 加上能够判别维度之间的因果关系以及学生满意度对学生抱怨、学生忠诚等因变量 的影响，本文选用该模型作为基础模型。由于校区后勤服务形象代表了师生对校区后勤服务 的全体性认识, 是学生选择前往校区学习的重要因素之一, 具有较高的重要性及相关性, 因 此加入该潜变量。

模型最终包含 7 个潜变量, 分别是:

后勤服务形象: 指师生对 PESI 校区后勤服务形象的群体性认识, 主要包括后勤形象及师 生口碑。

学生预期：指学生在赴法国留学前对校区后勤服务质量的估计，包括对学习生活环境、 设施的预期、对校区后勤服务可靠性的预期以及个人在国外留学期间的适应性预期 3 个观察 变量。

感知质量：指学生在体验校区后勤服务后对其质量的实际感受，包括对校区学习、生活 软件硬件服务质量及后勤服务人员反馈速度的体验。

感知价值：体现了学生在综合校区后勤服务的质量和价格以后对其利益的主观感受，包 括“给定价格条件下对后勤服务质量的感受”和“给定后勤服务质量条件下对价格的感受”2 个 观察变量。

总体满意度：最终得到的学生对后勤服务的满意度指数, 共包含 3 个观察变量。其中绝 对满意度指实际感受与理想后勤服务产品的差距, 差距越小, 满意度越高; 与预期相比的满 意度主要决定了学生对后勤服务的总体满意度; 对后勤服务态度的满意度能够说明后勤服务 提供商及其工作人员的服务理念。

学生抱怨：包括学生对校区后勤服务的正式抱怨及非正式抱怨。由于高校是后勤服务提 供商的采购及沟通单位, 因此学生的正式抱怨主要集中在向学校管理人员投诉; 而非正式抱 怨主要通过向亲友同学及在社交网络中发表信息的渠道完成。

学生忠诚: 学生忠诚是模型中最终的因变量, 如果学生对校区后勤服务满意, 就会产生 对校区后勤服务的忠诚。由于学生再次回到校区重新体验后勤服务的可能性非常小，因此只 能通过假设重复购买及推荐购买可能性 2 个观察变量评价学生忠诚度。

提出以下研究假设:

H1a：后勤服务形象和学生预期正相关

$\mathrm{H} 1 \mathrm{~b}$ ：后勤服务形象和满意度正相关

H1c：后勤服务形象与学生忠诚正相关

$\mathrm{H} 2 \mathrm{a}$ : 学生预期与感知价值正相关

$\mathrm{H} 2 \mathrm{~b}$ : 学生预期与感知质量正相关

H2c：学生预期与满意度正相关

$\mathrm{H} 3 \mathrm{a}$ : 感知质量与感知价值正相关

$\mathrm{H} 3 \mathrm{~b}$ : 感知质量与满意度正相关 
$\mathrm{H} 4$ ：感知价值与满意度正相关

$\mathrm{H} 5 \mathrm{a}$ : 学生满意度与学生抱怨负相关

$\mathrm{H} 5 \mathrm{~b}$ : 学生满意度与学生忠诚正相关

H6 ：学生抱怨与学生忠诚负相关

\section{2 量表设计}

由于通过结构方程建立海外校区办学后勤满意度指标模型的文献较少, 没有适合本研究 的量表可以使用，因此在结合李思宇、田坤、王晓永等设计的关于旅游满意度及高等教育满 意度的量表基础上 ${ }^{[10-12]}$, 结合 PESI 实际提供的服务内容及在工作中出现问题设计问卷。问卷 经过了学校管理人员及学生代表前测, 以确保内容效度; 通过预测试, 计算题项的分辨力, 删除分辨力弱的题项, 确保计算信度, 形成了最终的量表。潜变量与测量指标如表 1 所示, 其中后勤服务形象为潜自变量 (外生变量), 其他 6 个潜变量为潜因变量 (内生变量), 测量 变量中除学生抱怨题项采用二项量表以外，其他变量均采用 likert 10 级量表。

表 1: 调查问卷各变量测量指标

\begin{tabular}{|c|c|c|}
\hline 潜变量 & 题数 & 测量指标 \\
\hline \multirow{2}{*}{ 后勤服务形象（LQI） } & \multirow{2}{*}{2} & 校区形象（LQI1） \\
\hline & & 教师、同学口碑（LQI2） \\
\hline \multirow{3}{*}{ 学生预期（SE） } & \multirow{3}{*}{3} & 学习生活条件期望（SE1） \\
\hline & & 个人适应期望（SE2） \\
\hline & & 后勤服务可靠性期望（SE3） \\
\hline \multirow{8}{*}{ 感知质量（SQ） } & \multirow{8}{*}{8} & 住宿质量（SQ1） \\
\hline & & 生活服务, 包括供水供热供暖洗衣等 (SQ2) \\
\hline & & 保洁卫生（SQ3） \\
\hline & & 食堂供餐（SQ4） \\
\hline & & 体育锻炼场地及器材（SQ5） \\
\hline & & 学习环境（SQ6） \\
\hline & & 图书资料资源（SQ7） \\
\hline & & 后勤反馈速度（SQ8） \\
\hline \multirow{2}{*}{ 感知价值（SP） } & \multirow{2}{*}{2} & 现有服务条件下价格是否合理（SP1） \\
\hline & & 现有价格前提下对服务是否满意（SP2） \\
\hline \multirow{3}{*}{ 学生满意度（MS） } & \multirow{3}{*}{3} & 对后勤服务绝对满意度（MS1） \\
\hline & & 和预期相比相对满意度（MS2） \\
\hline & & 对服务态度的满意度（MS3） \\
\hline \multirow{3}{*}{ 学生抱怨（SC） } & \multirow{3}{*}{3} & 向家长和朋友抱怨（SC1） \\
\hline & & 社交软件上抱怨 (SC2) \\
\hline & & 向学校管理人员投诉 (SC3) \\
\hline \multirow{2}{*}{ 学生忠诚（SL） } & \multirow{2}{*}{2} & 是否向同学推荐 (SL1) \\
\hline & & 若有重新 1 次选择机会, 是否愿意再来 (SL2) \\
\hline
\end{tabular}

\section{3 数据收集}

本研究的问卷调查数据采集于 2018 年 7 月-10 月，采用网络发送问卷的形式，问卷说明 了本次调查为纯学术研究, 以减少填写误差。问卷对象涵盖了 BISU 大学和 BSU 大学 2 所高 校派出至法国 PESI 校区学习的学生, 共回收问卷 127 份, 其中有效问卷 125 份, 有效率为 $98.4 \%$ 。样本结构见表 2 。 
表 2: 样本结构

\begin{tabular}{|c|c|c|}
\hline 项目 & 类别 & 人数 \\
\hline \multirow{2}{*}{ 所属高校 } & BISU & 111 \\
\hline \multirow{3}{*}{ 所在年级 } & BSU & 14 \\
\cline { 2 - 3 } & 大二 & 64 \\
\cline { 2 - 3 } & 大三 & 47 \\
\cline { 2 - 3 } & 大四 & 14 \\
\hline \multirow{2}{*}{ 性别 } & 男 & 15 \\
\cline { 2 - 3 } & 女 & 110 \\
\hline
\end{tabular}

\section{4. 计算模型的建立和修正}

\section{1 估计方法选择}

在对各题项数据的正态性进行 Shapiro-Wilk 检验后, 发现在 5\%的显著性水平上, 所有题 项的正态性分布假设均被拒绝, 因此无法选用 Linear Structural RELationships (LISREL) 估

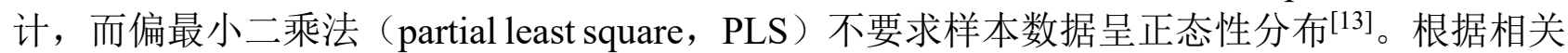
资料, 结构方程模型建模时样本量至少为顾客满意度模型中具有最多结构路径指向的隐变量 的路径数的十倍, 更弱一些的限制类似于多元回归,也应至少五倍 ${ }^{[14]}$; 且 PLS 估计时样本量最 小推荐 100 个; 最好在 230 个以上 ${ }^{[15]}$ 。本研究潜变量中题项最多的是感知质量, 共有 8 个题 项, 本研究共 125 个样本, 符合 PLS 估计的样本量要求。综上所述, 本研究选用 PLS 估计, 使用 SmarTPLS3.0 软件计算。

\section{2 信度和效度分析}

\section{2 .1 信度分析}

在海外校区后勤服务满意度评价中, 信度指的是调查数据所反映学生满意程度的可靠性。 学生对后勤服务的感受趋向于一致, 随机因素的影响越小, 测评数据就越客观, $\alpha$ 值就会越 大。如表 3 所示, 本研究中潜变量 $\alpha$ 值均大于 0.7 , 说明本研究构建的后勤服务满意度评价模 型潜变量可信度高 ${ }^{[16]}$ 。通过对综合信度的分析可以测度模型表现出较好的内部一致性, 根据 前人研究建议, 综合信度应大 $0.7^{[17]}$ 。本研究中各潜变量的综合信度均大于 0.836 , 表明潜变 量的变化能够解释对应测量工具至少 $83.6 \%$ 的变化, 说明测量模型内部一致性较好, 描述是 可信的。所有潜变量的平均提取方差 (AVE) 超过 0.63, 大于 Fornell 和 Larcker (1981) 提出 的临界值 0.5 , 说明显变量很好的解释了潜变量, 具有较好的收玫效度 ${ }^{[18]}$ 。

表 3: 信度和效度

\begin{tabular}{|c|c|c|c|c|}
\hline 潜变量 & $\mathrm{R}^{2}$ & 科隆巴赫 $\alpha$ & 综合信度 & AVE \\
\hline 后勤服务形象 & - & 0.891 & 0.948 & 0.901 \\
\hline 学生满意度 & 0.887 & 0.927 & 0.953 & 0.872 \\
\hline 学生抱怨 & 0.530 & 0.707 & 0.836 & 0.630 \\
\hline 学生预期 & 0.445 & 0.816 & 0.890 & 0.732 \\
\hline 学生忠诚 & 0.761 & 0.911 & 0.957 & 0.918 \\
\hline 感知价值 & 0.733 & 0.980 & 0.990 & 0.980 \\
\hline 感知质量 & 0.230 & 0.926 & 0.940 & 0.661 \\
\hline
\end{tabular}

\subsection{2 验证性因子分析（CFA）}

通过验证性因子分析, 可以确定题项是否属于潜变量的范畴，如表 4 所示，除个人适应 期望外, 各题项的因子负荷均大于 0.7 , 且大于与其他因子的交叉载荷, 说明题项均属于其潜 变量范畴, 潜变量之间具有较好的区分效度。个人适应期望反映了学生对于在海外校区的生 
活能否适应的预判, 从定义上判断属于学生预期潜变量, 其在学生期望的因子负荷量为 0.693 , 非常接近 0.7 且超出交叉载荷 0.3 以上, 因此将该题项保留, 仍然列入学生预期潜变量。

表 4：验证性因子分析

\begin{tabular}{|c|c|c|c|c|c|c|c|c|c|}
\hline \multirow{2}{*}{ 题项 } & \multirow{2}{*}{ 均值 } & 标准 & \multicolumn{9}{|c|}{ 差 } & LQI & SE & SP & SQ & MS & SC & SL \\
\hline LQ1 & 7.568 & 1.932 & $\mathbf{0 . 9 5 4}$ & 0.636 & 0.552 & 0.611 & 0.554 & -0.486 & 0.429 \\
\hline LQ12 & 7.496 & 2.074 & $\mathbf{0 . 9 4 5}$ & 0.630 & 0.498 & 0.496 & 0.459 & -0.392 & 0.384 \\
\hline SE1 & 7.392 & 2.008 & 0.672 & $\mathbf{0 . 9 0 2}$ & 0.397 & 0.413 & 0.335 & -0.316 & 0.315 \\
\hline SE2 & 8.136 & 1.531 & 0.285 & $\mathbf{0 . 6 9 3}$ & 0.235 & 0.335 & 0.225 & -0.200 & 0.248 \\
\hline SE3 & 7.704 & 1.955 & 0.662 & $\mathbf{0 . 9 5 1}$ & 0.445 & 0.472 & 0.390 & -0.347 & 0.345 \\
\hline SP1 & 6.568 & 2.528 & 0.548 & 0.440 & $\mathbf{0 . 9 9 0}$ & 0.843 & 0.917 & -0.697 & 0.850 \\
\hline SP2 & 6.512 & 2.657 & 0.549 & 0.422 & $\mathbf{0 . 9 9 0}$ & 0.852 & 0.924 & -0.700 & 0.861 \\
\hline SQ1 & 6.664 & 2.462 & 0.483 & 0.395 & 0.817 & $\mathbf{0 . 8 8 0}$ & 0.810 & -0.594 & 0.772 \\
\hline SQ2 & 6.968 & 2.342 & 0.535 & 0.434 & 0.674 & $\mathbf{0 . 8 0 4}$ & 0.670 & -0.430 & 0.622 \\
\hline SQ3 & 7.200 & 2.148 & 0.534 & 0.416 & 0.739 & $\mathbf{0 . 8 6 2}$ & 0.739 & -0.582 & 0.662 \\
\hline SQ4 & 6.688 & 2.223 & 0.506 & 0.399 & 0.700 & $\mathbf{0 . 8 5 3}$ & 0.688 & -0.567 & 0.651 \\
\hline SQ5 & 7.000 & 2.352 & 0.385 & 0.352 & 0.594 & $\mathbf{0 . 7 5 1}$ & 0.610 & -0.437 & 0.575 \\
\hline SQ6 & 7.264 & 2.029 & 0.350 & 0.373 & 0.677 & $\mathbf{0 . 8 1 9}$ & 0.668 & -0.420 & 0.689 \\
\hline SQ7 & 5.736 & 2.294 & 0.416 & 0.402 & 0.541 & $\mathbf{0 . 7 2 8}$ & 0.553 & -0.380 & 0.537 \\
\hline SQ8 & 6.824 & 2.700 & 0.571 & 0.357 & 0.770 & $\mathbf{0 . 7 9 4}$ & 0.829 & -0.624 & 0.728 \\
\hline MS1 & 6.968 & 2.423 & 0.562 & 0.424 & 0.934 & 0.867 & $\mathbf{0 . 9 5 3}$ & -0.667 & 0.878 \\
\hline MS2 & 6.760 & 2.644 & 0.430 & 0.291 & 0.889 & 0.835 & $\mathbf{0 . 9 4 9}$ & -0.697 & 0.837 \\
\hline MS3 & 7.672 & 2.327 & 0.509 & 0.350 & 0.775 & 0.710 & $\mathbf{0 . 8 9 9}$ & -0.679 & 0.714 \\
\hline SC1 & 0.512 & 0.502 & -0.443 & -0.296 & -0.648 & -0.643 & -0.666 & $\mathbf{0 . 8 4 9}$ & -0.592 \\
\hline SC2 & 0.224 & 0.419 & -0.279 & -0.244 & -0.537 & -0.401 & -0.508 & $\mathbf{0 . 7 7 5}$ & -0.524 \\
\hline SC3 & 0.184 & 0.389 & -0.372 & -0.285 & -0.480 & -0.425 & -0.547 & $\mathbf{0 . 7 5 5}$ & -0.437 \\
\hline SL1 & 6.520 & 2.548 & 0.482 & 0.382 & 0.866 & 0.811 & 0.881 & -0.657 & $\mathbf{0 . 9 6 3}$ \\
\hline SL2 & 7.024 & 2.598 & 0.332 & 0.297 & 0.786 & 0.740 & 0.782 & -0.599 & $\mathbf{0 . 9 5 3}$ \\
\hline
\end{tabular}

注: 粗体数字为测量变量与对应潜变量的因子载荷量

\section{3 结构方程建模}

将原始数据导入 SmartP1s3.0 软件, 并按照量表设计的潜变量和题项关系建立模型, 如图 1 所示。

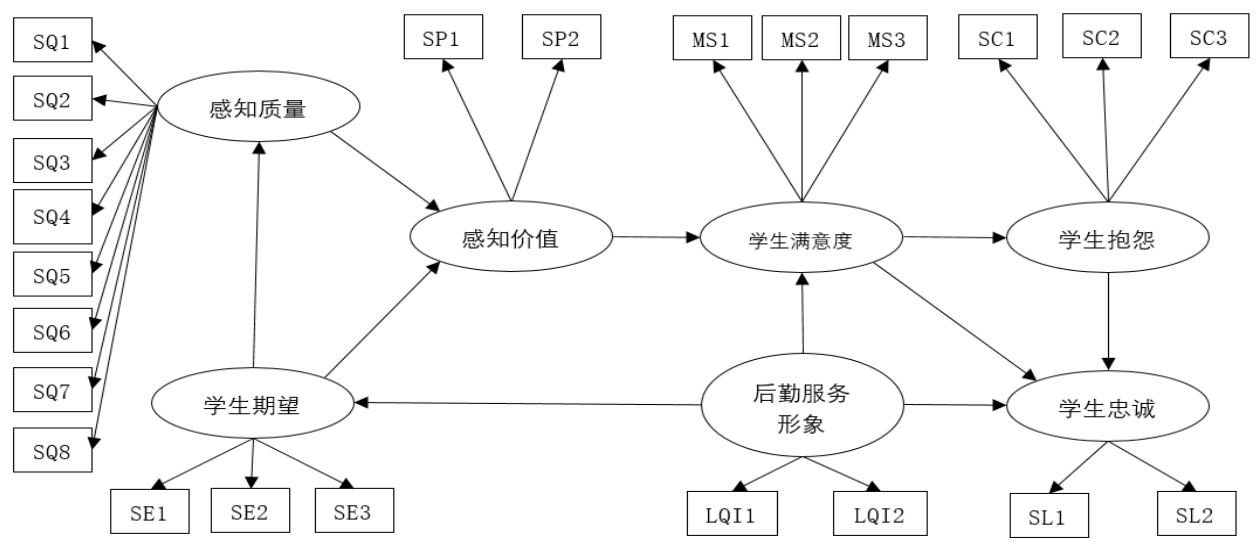

图 1: 学生对海外校区后勤服务满意度指数模型 


\subsection{1 模型评价}

$\mathrm{R}^{2}$ 为模型中潜变量与其相应解释潜变量之间因子负荷和相关系数的乘积之和, 表示解释

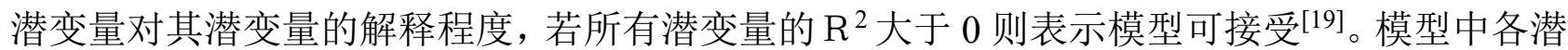
变量的 $\mathrm{R}^{2}$ 均大于 0 , 且总体满意度的 $\mathrm{R}^{2}$ 达到 0.927 , 说明学生总体满意度被后勤服务形象、 学生预期、质量感知、价格感知解释的比例达到 $92.7 \%$, 符合要求。

通过在 SMARTPLS 软件中进行 bootstrapping 计算验证路径系数的显著性，如表 5 所示， 有 5 条路径的 $\mathrm{T}$ 值小于 1.96 , 在 $\mathrm{p}<0.05$ 的水平上不显著, 说明校区后勤形象对学生综合满意 度及学生忠诚没有直接相关, 学生预期对价值感知及学生满意度没有直接影响, 学生抱怨也 不能直接影响学生忠诚。

表 5: 路径假设检验

\begin{tabular}{|c|c|c|c|}
\hline 潜变量路径 & 参数估计 & $\mathrm{T}$ 值 & $\mathrm{P}$ 值 \\
\hline 学生满意度<- 后勤服务形象 & 0.038 & 0.722 & 0.470 \\
\hline 学生预期<- 后勤服务形象 & 0.667 & 9.113 & $* * *$ \\
\hline 学生忠诚<- 后勤服务形象 & -0.058 & 0.871 & 0.384 \\
\hline 学生抱怨 <- 学生满意度 & -0.728 & 17.404 & $* * *$ \\
\hline 学生忠诚<- 学生满意度 & 0.859 & 13.038 & $* * *$ \\
\hline 学生忠诚<- 学生抱怨 & -0.059 & 0.833 & 0.405 \\
\hline 学生满意度<- 学生预期 & -0.086 & 1.849 & 0.065 \\
\hline 感知价值<- 学生预期 & 0.032 & 0.543 & 0.587 \\
\hline 感知质量 <- 学生预期 & 0.479 & 6.632 & $* * *$ \\
\hline 学生满意度 <- 感知价值 & 0.713 & 10.200 & $* * *$ \\
\hline 学生满意度<- 感知质量 & 0.274 & 3.429 & $* * *$ \\
\hline 感知价值<- 感知质量 & 0.840 & 21.188 & $* * *$ \\
\hline
\end{tabular}

注：***代表在 $1 \%$ 的水平上显著

\subsection{2 结构模型修正}

由于初始模型中有 5 条路径没有通过显著性检验，因此对结构模型进行修正，删除未通 过显著性检验的路径。如表 6 所示，修正后模型的 $\mathrm{R}^{2}$ 仍然大于 0 ，且学生综合满意度达到 0.883 , 被后勤服务形象、学生预期、质量感知、价格感知解释的比例仍有 $88.3 \%$, 说明修正 后的模型可信度高。将样本分为 7 组通过 Blindfolding 计算, 所有潜变量的 $\mathrm{Q}^{2}$ 均大于 0 , 说 明模型具备良好的预测性 ${ }^{[20]}$ 。

表 6: 修正后模型 $\mathrm{R}^{2}$ 和 $\mathrm{Q}^{2}$

\begin{tabular}{|c|c|c|c|}
\hline \multirow{2}{*}{ 潜变量 } & \multirow{2}{*}{$\mathrm{R}^{2}$} & \multicolumn{2}{|c|}{$\mathrm{Q}^{2}$} \\
\cline { 3 - 4 } & & 测量模型共同性 & 结构模型重叠性 \\
\hline 后勤服务形象 & - & 0.531 & - \\
\hline 学生满意度 & 0.883 & 0.649 & 0.719 \\
\hline 学生抱怨 & 0.532 & 0.273 & 0.312 \\
\hline 学生预期 & 0.447 & 0.456 & 0.294 \\
\hline 学生忠诚 & 0.758 & 0.560 & 0.660 \\
\hline 感知价值 & 0.732 & 0.675 & 0.683 \\
\hline 感知质量 & 0.230 & 0.532 & 0.141 \\
\hline
\end{tabular}

如表 7 所示，修正后模型中各路径系数以及因子载荷参数的 $\mathrm{T}$ 值均大于 1.96 ，显著性均 通过检验。 
表 7：路径系数、因子载荷参数估计及假设检验

\begin{tabular}{|c|c|c|c|}
\hline 路径系数/因子载荷 & 参数估计 & $\mathrm{T}$ 值 & $\mathrm{P}$ 值 \\
\hline 学生预期〈- 后勤服务形象 & 0.668 & 8.980 & $* * *$ \\
\hline 感知质量<- 学生期望 & 0.479 & 6.700 & $* * *$ \\
\hline 感知价值 <- 感知质量 & 0.856 & 35.769 & $* * *$ \\
\hline 学生满意度<- 感知价值 & 0.712 & 9.937 & $* * *$ \\
\hline 学生满意度<- 感知质量 & 0.255 & 3.480 & $* * *$ \\
\hline 学生抱怨 <- 学生满意度 & -0.729 & 16.800 & $* * *$ \\
\hline 学生忠诚<- 学生满意度 & 0.871 & 48. 209 & $* * *$ \\
\hline LQI1<- 后勤服务形象 & 0.950 & 71.114 & $* * *$ \\
\hline LQI2<- 后勤服务形象 & 0.949 & 92.847 & $* * *$ \\
\hline SE1<- 学生期望 & 0.905 & 28.661 & $* * *$ \\
\hline SE2<- 学生期望 & 0.688 & 7.052 & $* * *$ \\
\hline SE3<- 学生期望 & 0.951 & 89.167 & $* * *$ \\
\hline SQ1<- 感知质量 & 0.880 & 49.846 & $* * *$ \\
\hline SQ2<- 感知质量 & 0.804 & 16.949 & $* * *$ \\
\hline SQ3<- 感知质量 & 0.862 & 37.637 & $* * *$ \\
\hline SQ4<- 感知质量 & 0.853 & 28.136 & $* * *$ \\
\hline SQ5<- 感知质量 & 0.751 & 11.920 & $* * *$ \\
\hline SQ6<- 感知质量 & 0.819 & 28.100 & $* * *$ \\
\hline SQ7<- 感知质量 & 0.728 & 12.794 & $* * *$ \\
\hline SQ8<- 感知质量 & 0.794 & 17.573 & $* * *$ \\
\hline SP1 <- 感知价值 & 0.990 & 270.153 & $* * *$ \\
\hline SP2<- 感知价值 & 0.990 & 293.835 & $* * *$ \\
\hline MS1 <- 学生满意度 & 0.953 & 79.553 & $* * *$ \\
\hline MS2<- 学生满意度 & 0.949 & 46. 018 & $* * *$ \\
\hline MS3<- 学生满意度 & 0.899 & 29.460 & $* * *$ \\
\hline SC1 <- 学生抱怨 & 0.850 & 35.988 & $* * *$ \\
\hline SC2<- 学生抱怨 & 0.761 & 14.787 & $* * *$ \\
\hline SC3<- 学生抱怨 & 0.767 & 15.514 & $* * *$ \\
\hline SL1 <- 学生忠诚 & 0.963 & 117.181 & $* * *$ \\
\hline SL2<- 学生忠诚 & 0.953 & 55.858 & $* * *$ \\
\hline
\end{tabular}

注：***代表在 $1 \%$ 的水平上显著

综上, 本研究最终建立的结构方程模型如图 2 所示, 假设的验证结果如表 8 所示。 


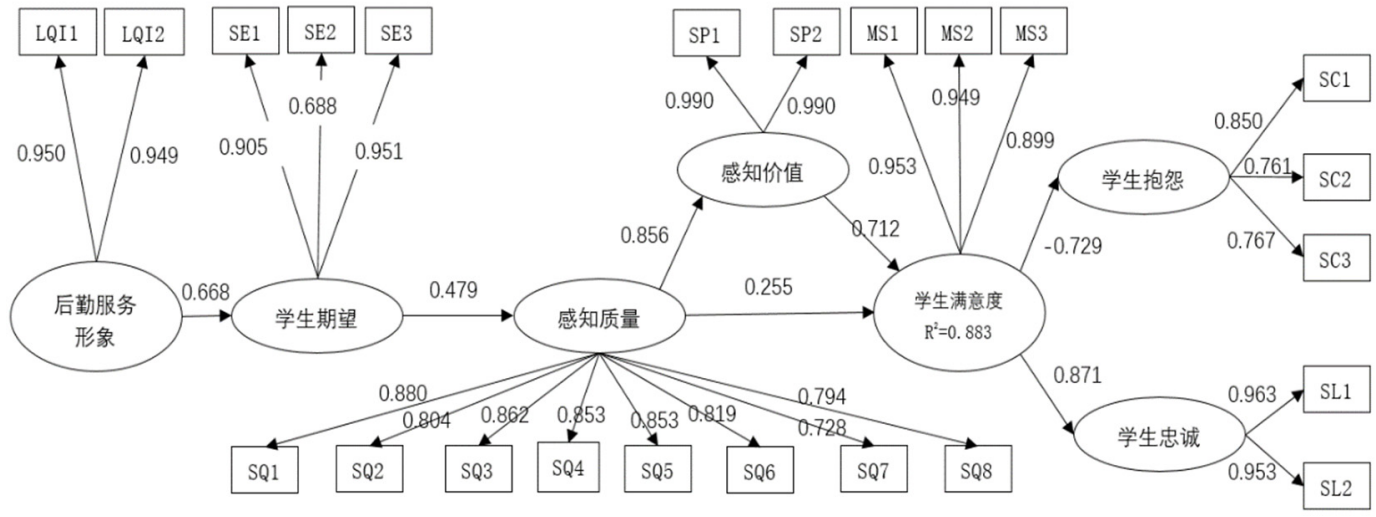

图 2: 修正后的满意度指数模型

表 8: 模型的假设检验结果

\begin{tabular}{|l|c|c|}
\hline \multicolumn{1}{|c|}{ 假设 } & $\mathrm{T}$ 值 & 支持与否 \\
\hline $\mathrm{H} 1 \mathrm{a}:$ 后勤服务形象和学生预期正相关 & 9.113 & 是 \\
\hline $\mathrm{H} 1 \mathrm{~b}:$ 后勤服务形象和学生满意度正相关 & 0.722 & 否 \\
\hline $\mathrm{H} 1 \mathrm{c}:$ 后勤服务形象与学生忠诚正相关 & 0.871 & 否 \\
\hline $\mathrm{H} 2 \mathrm{a}:$ 学生预期与感知价值正相关 & 0.543 & 否 \\
\hline $\mathrm{H} 2 \mathrm{~b}:$ 学生预期与感知质量正相关 & 6.632 & 是 \\
\hline $\mathrm{H} 2 \mathrm{c}:$ 学生预期与学生满意度正相关 & 1.849 & 否 \\
\hline $\mathrm{H} 3 \mathrm{a}:$ 感知质量与感知价值正相关 & 21.188 & 是 \\
\hline $\mathrm{H} 3 \mathrm{~b}:$ 感知质量与学生满意度正相关 & 3.429 & 是 \\
\hline $\mathrm{H} 4$ : 感知价值与学生满意度正相关 & 10.200 & 是 \\
\hline $\mathrm{H} 5 \mathrm{a}:$ 学生满意度与学生抱怨负相关 & 17.404 & 是 \\
\hline $\mathrm{H} 5 \mathrm{~b}:$ 学生满意度与学生忠诚正相关 & 13.038 & 是 \\
\hline $\mathrm{H} 6$ : 学生抱怨与学生忠诚负相关 & 0.833 & 否 \\
\hline
\end{tabular}

\section{4 中介效应}

通过 SmarTPLS 软件中 bootstrapping 算法计算中介效应，如果 $\mathrm{T}$ 值大于 1.96 时则为显 著，表明中介效应存在 ${ }^{[21]}$ 。虽然后勤服务形象、学生期望对学生满意度没有直接效应，但是 通过中介效应检测可以发现这些潜变量是否能够间接影响学生的满意度指数, 此外, 感知质 量对学生满意度有直接影响, 也可以通过感知价值产生间接影响, 通过计算综合效应来对比 感知质量与感知价值对学生满意度的综合影响。中介效应及综合效应的计算如表 9 所示。

表 9：中介效应及综合效应

\begin{tabular}{|l|c|c|c|c|c|c|}
\hline \multirow{2}{*}{ 路径 } & \multirow{2}{*}{ 直接效应 } & \multicolumn{3}{|c|}{ 中介效应 } & \multirow{2}{*}{ 综合效应 } & \multirow{2}{*}{ 效应类型 } \\
\cline { 3 - 5 } & & 路径系数 & $\mathrm{T}$ 值 & $\mathrm{P}$ 值 & & \\
\hline 学生满意度 $<-$ 后勤服务形象 & - & 0.277 & 4.626 & $* * *$ & 0.277 & 只有中介效应 \\
\hline 学生满意度 $<-$ 学生期望 & - & 0.414 & 6.462 & $* * *$ & 0.414 & 只有中介效应 \\
\hline 学生满意度 $<-$ 感知质量 & 0.255 & 0.609 & 10.045 & $* * *$ & 0.864 & 互补型中介 \\
\hline 学生满意度 $<-$ 感知价值 & 0.712 & - & - & - & 0.712 & 只有直接效应 \\
\hline
\end{tabular}

注: $* * *$ 代表在 $1 \%$ 的水平上显著

\section{5 学生对PESI提供的后勤服务满意度指数得分}

学生满意度指数计算公式为 ${ }^{[22]}$ : 


$$
\mathrm{SSI}=\frac{E\left[M S_{3}\right]-\operatorname{Min}\left\{M S_{3}\right\}}{\operatorname{Max}\left\{M S_{3}\right\}-\operatorname{Min}\left\{M S_{3}\right\}} \times 100 \%
$$

其中 $\mathrm{E}\left[M S_{3}\right]=\sum_{i=1}^{3} w_{i} \cdot m s_{i}, \quad \operatorname{Min}_{2}\left\{M S_{3}\right\}=\sum_{i=1}^{3} w_{i} \cdot \min \left\{m s_{i}\right\}, \quad \operatorname{Max}\left\{M S_{3}\right\}=\sum_{i=1}^{3} w_{i} \cdot$ $\max \left\{m s_{i}\right\}$ 。

式中 $M S_{3}$ 为学生满意度的潜变量, 显变量个数为 $3, \mathrm{E}\left[M S_{3}\right]$ 是学生满意度的期望的得分, $\operatorname{Min}\left\{M S_{3}\right\}$ 是学生满意度的最低得分, $\operatorname{Max}\left\{M S_{3}\right\}$ 是学生满意度的最高得分。

由于采用 10 级量表, 所以 $\operatorname{Min}\left[M S_{3}\right]=1, \operatorname{Max}\left[M S_{3}\right]=10, \mathrm{SSI}$ 计算公式演变为:

$$
\mathrm{SSI}=\frac{\sum_{i=1}^{3} w_{i} \cdot \overline{m s_{l}}-\sum_{i=1}^{3} w_{i}}{9 \sum_{i=1}^{3} w_{i}} \times 100 \%
$$

其中 $w_{i}$ 为显变量权重, $\overline{m s_{l}}$ 为第 $\mathrm{i}$ 个显变量的均值, 学生满意度显变量均值和权重见表 10 , 带入公式得出 SSI=68.0。说明学生对 PESI 提供的后勤服务满意度一般, 校区后勤服务水平还 有较大的提升空间。

表 10: 学生满意度显变量均值与权重

\begin{tabular}{|c|c|c|}
\hline 显变量 & 权重 & 均值 \\
\hline MS1 & 0.953 & 6.968 \\
\hline MS2 & 0.949 & 6.76 \\
\hline MS3 & 0.899 & 7.672 \\
\hline
\end{tabular}

\section{5. 讨论}

在后勤服务形象的维度中，学生对校区形象的认识及口碑的因子负荷量基本相等，这可 能是由于校区地处法国, 学生缺少深入了解校区的途径, 加上对师生的信任, 因此对校区后 勤服务形象的认识主要来源于口碑。学校在招生宣传过程中, 要注意引导师生客观真实的评 价校区后勤设施及服务; 此外，应该通过提升后勤服务水平、优化服务态度提升师生对后勤 保障系统的口碑。

在学生期望维度中，后勤服务可靠性的影响最大，因子负荷量最高；个人适应期望的因 子负荷量最低, 可能因为学生做出留学决策时, 对学习生活习惯及环境的变化有一定程度的 心理准备, 对能够适应当地文化有较强的自信。校区后勤服务是教学活动顺利开展、师生生 活正常保障的基础, 因此要及时的解决后勤服务中出现的问题, 避免在学生群体中产生后勤 服务靠不住的印象, 影响学生的满意度。

在感知价值维度中，对价格和服务的满意度的因子负荷量基本相等，说明价格和服务质 量的满意度较为一致, 没有出现偏差。在感知质量维度中, 住宿质量的因子负荷量最高, 是 学生最重视的后勤服务内容, 保洁卫生及食堂供餐紧随其后。图书资料资源的因子负荷量最 低, 这可能是因为随着信息技术的迅速发展, 学生的阅读习惯倾向于电子化、网络化, 纸质 书籍的重要性下降明显, 此外, 限于语言水平, 学生阅读法语书籍难度较高, 对书籍的需求 并不旺盛。

在总体满意度维度中，学生对后勤服务的绝对满意及与预期相比的相对满意度的比重更 大，而对服务态度满意度相对来说影响较小，这可能是由于校区后勤服务人员不仅仅是服务 提供者, 也是实现管理育人、服务育人的教育工作者, 学生在心理上处于相对弱势的地位, 对服务态度的关注较其他类型消费者更低。

在学生抱怨维度中，向家长和朋友抱怨的因子负荷量最高, 且有 $51.2 \%$ 的学生曾向家长 或者朋友抱怨, 而在社交软件上抱怨的仅占 $22.4 \%$, 曾向学校管理教师投诉的仅占 $18.4 \%$, 说 明学生主要的抱怨途径是家长和朋友。在学生忠诚维度中, 学生对推荐同学来校区学习的可 能性低于自己前往, 说明仍疑虑同学能否适应校区的后勤服务。然而推荐同学的因子负荷量 更高，更能代表学生对校区服务的忠诚程度。 
通过对模型中各路径的分析，发现影响后勤服务满意度的因素，指导学校后勤管理部门 及后勤服务提供商如何提升学生的满意度。质量感知和价格感知均与学生满意度正向相关, 说明可以通过提升服务质量及后勤产品性价比来提升学生的满意度; 质量感知的直接效应比 价值感知低, 但是综合效应更高, 说明学生对后勤的满意度受后勤产品的性价比的直接影响 更多, 但是通过提升服务质量也能够提升产品性价比, 是提升满意度的最重要因素。后勤服 务形象对学生满意度及学生忠诚的直接影响并不显著, 说明校区后勤服务形象宣传不能直接 提升学生的满意度; 但是间接效应显著, 说明做好校区后勤形象宣传工作，提升后勤服务形 象, 可以促进学生更加深入的了解校区后勤服务体系, 通过让学生建立合理的期望, 提升质 量感知和价值感知, 间接的提升学生对后勤的满意度。学生预期对感知质量的直接影响显著, 对感知价值及学生满意度的直接影响不显著, 但是间接效应显著, 说明虽然学生的预期对其 满意度并无直接影响, 但是供应商应深入了解学生对后勤服务需求, 相应调整后勤服务策略, 提供性价比更加合理的后勤产品, 可以间接提升学生的满意度。学生对后勤的满意度指标会 直接影响学生对后勤产品的抱怨以及学生对校区的忠诚度, 然而学生抱怨对学生忠诚的直接 影响并不显著, 说明学生的抱怨不会直接影响其忠诚度, 因此后勤服务人员在面对学生抱怨 时，应注意解决相关服务问题，不要因为学生抱怨而对学生产生不满心理。

\section{6. 结论}

本研究通过实证分析，在 AESI 的基础上，构建了学生对企业在海外校区提供的后勤服 务满意度指数模型, 为高校在未来赴海外办学中评价后勤供应商提供了评价工具。该模型的 信度、效度、以及解释度均通过了科学验证, 包括校区后勤服务形象、学生期望、质量感知、 价值感知、学生满意度、学生抱怨以及学生忠诚共 7 个潜变量和 23 个显变量。

研究的结论可在高校海外办学中后勤管理部门制定后勤政策以及后勤供应商制定服务策 略时提供理论依据, 并提出建议: 为提升学生对海外校区后勤服务的满意度, 应首先提升服 务质量, 提供高性价比的后勤服务产品; 其次通过各种方式深入了解海外校区学生对后勤服 务的需求, 有针对性的调整服务重心; , 此外还应做好校区后勤服务的宣传工作, 加强学生对 校区后勤服务的了解。

本文基于实证数据，对测量模型也进行了一定程度上的修正和检验，仍然存在一些局限 性：由于中国高校赴海外办学仍属于新兴事务, 相关研究较少, 因此本研究借鉴消费者满意 度模型来建立模型。然而高校后勤具备公益性特征, 并非完全意义上的消费产品, 学生也不 是完全意义上的消费者, 因此模型可能有所缺陷。此外, PESI 校区运营时间较短, 学生较少, 因此本研究可以获取的样本较少, 样本分布不均衡, 可能影响估计的精确性。

在未来的研究中，应根据中国高校后勤的特征及海外办学的实际情况，基于适用的理论 建立更加完善的模型; 此外, 还可以通过时间积累, 收集更多的样本, 提升样本多样性及样 本结构的合理性，提升估计的精确度。

\section{致谢}

本文为北京第二外国语学院 2018 年校级科研项目（XJGH18A001）的阶段性成果之一。

\section{References}

[1] Lin Wenxin, Discussion on University Logistics Management Mode Based on Customer Satisfaction, University Logistics Support, vol. 03, pp. 33-35, 2014.

[2] Feng Baojun and Zhang Sipeng, Research on the Service Outsourcing Model for University Logistics Socialization Reform:An Example of University D, vol. 07, pp. 47-57, 2014. 
[3] Jia Bin and Wang yi, The Construction of Evaluation System of Logistics Service Satisfaction Index in Higher Vocational Colleges under the New Situation-Taking Yangzhou Industrial Vocational and Technical College as an Example, Brand, vol. 10, pp. 50-52, 2014.

[4] Wang Wenya and Chen Binbin, Research on the Satisfaction Survey of University Logistics Service-Taking the Logistics of Peking University Medical Department as an Example, University Logistics Research, vol. 08, pp. 26-28, 2016.

[5] Zhong Yi and Zhang Haijun, Research on Student Satisfaction Evaluation Model of Logistics Service, University Logistics Research, vol. 08, pp. 50-52, 2017.

[6] Sun Tao, Research on University Logistics Service Evaluation Based on Teachers and Students Satisfaction, Value Engineering, vol. 11, pp. 174-175, 2018.

[7] Tian Liang and Tian Wenfang, An Analysis of the Satisfaction Survey of College Canteens in Xinjiang--Taking Tarim University as an Example, Science \& Technology Vision, vol. 34, pp. 40-41, 2014.

[8] Liu Xueping, Evaluation of Student Apartment Management Satisfaction Based on SEM Model, University Logistics Research, vol. 01, pp. 33-36, 2014.

[9] Xue Liang, Research on University Logistics Management Mode Based on Customer Satisfaction, New Campus, vol. 02, pp. 13-13, 2018.

[10] Li Siyu, The Analysis on the Satisfaction of Tourists in Guilin Based on the Structural Equation Model, Guangxi Normal University, 2017.

[11] Tian Kun, On the Evaluation Index System of Examinees Satisfaction of University Enrollment Service Based on ACSI, Xianstan University, 2017.

[12] Measurement Method and EmPirical Research on the Evaluation of Educational Satisfaction of Postgraduates in Heilongjiang Province, Harbin Engineering University, 2011.

[13] Cui bin and Zhang Yawei, Study on the Satisfaction Formation Mechanism of College Students' Entrepreneurship Education-Based on the PLS Structural Equation Model, China University Students Career Guide, vol. 22, pp. 55-59, 2013.

[14] Liang Yan, Research on Several Issues of Customer Satisfaction Index, Statistical Research, vol. 20, pp. 52-56, 2013.

[15] Liang Yan and Jin Yongjin, The Sample Size Study of the Customer Satisfaction Model, Statistical Research, vol. 24, pp. 68-74, 2007.

[16] Lee J. Cronbach, Coefficient alpha and the internal structure of tests, Psychometrika, vol. 16, pp. 297-334, 1951.

[17] Jin Yan, Construction of 3PL Customer Satisfaction Evaluation Model Based on SEM, Journal of Huaihai Institute of Technology(Natural Science Edition), vol. 02, pp. 75-80, 2014.

[18] Jiang Dongming and Wu Weihua, Research on the cleanliness of RMB banknotes in circulation, Financial Perspectives Journal, vol. 08, pp. 45-49, 2014.

[19] Zhao Fuqiang, Liu Jinlan, Research of Customer Satisfaction Measurement Based on PLS Path Modeling, Tianjin University, 2016.

[20] Tenenhaus M and Vinzi V E et al, PLS Path Modelin, Computational Statistics \& Data Analysis, vol. 48, pp. 159-205, 2005. 
[21] Preacher K J and Hayes A F, Asymptotic and Resampling Strategies for Assessing and Comparing Indirect Effects in Multiple Mediator Models, Behav Res Methods, vol. 40, pp. 879$891,2008$.

[22] Li Zhongdong and Guo Xiaomin, Research on Public Satisfaction of Food Safety Regulation Based on PLS-SEM, Journal of Shandong Technology and Business University, vol. 31, pp. 3542, 2017. 